\title{
Linearity and enhanced sensitivity of the Shipley AZ-1350B photoresist
}

\author{
A. C. Livanos, A. Katzir, J. B. Shellan, and A. Yariv
}

\begin{abstract}
The properties of the Shipley AZ-1350B positive photoresist used with the Shipley AZ-303A developer were investigated. It was found that the use of AZ-303A developer results in a significant improvement of the sensitivity and the linearity of the photoresist. The unexposed etch rate of the photoresist was $35 \AA \pm 5 \AA$ / sec. Gratings of high efficiency have been successfully fabricated using the above combination of photoresist and developer.
\end{abstract}

Current work in integrated optics requires the fabrication of relief grating structures on photoresist and the subsequent chemical or ion beam etching through the photoresist. ${ }^{1}$ If the period of the grating is to be less than $0.4 \mu \mathrm{m}$ the Shipley AZ-1350B photoresist is commonly used. The properties of this positive acting photoresist have been examined in detail, $, 2,3$ and it was found that the photoresist exhibits strong nonlinearity, especially for etch depth ranging from $0.05 \mu \mathrm{m}$ to $0.2 \mu \mathrm{m}$. Bartolini ${ }^{4,5}$ first showed that a different developer, namely the AZ-303A, used with the old AZ-1350 photoresist (now replaced by the 1350B) results in an unexposed resist etch rate of approximately $200 \AA / \mathrm{sec}$, removes the nonlinearity, and improves the sensitivity by a factor of 2 or 3 . Linearity and speed or sensitivity are always of practical interest. ${ }^{6,7}$ Norman and Singh ${ }^{8}$ have studied the characteristics of the AZ-1350J resist (which has replaced the old AZ-1350H) used with the AZ-303A developer and report results similar to the ones presented in Ref. 5 . It is the purpose of this paper to show that the AZ-303A developer can be used with the AZ-1350B photoresist resulting in improved sensitivity and linearity and unexposed resist etch rate of 35 $\pm 5 \AA / \mathrm{sec}$.

Bartolini ${ }^{5}$ has shown that for a positive acting photoresist the following relationship exists between etch depth $\Delta d$ and exposure $E$ (in units of energy per unit area):

$$
\Delta d=T\left[r_{1}-\Delta r \exp (-c E)\right],
$$

where $T$ is the development time in seconds, $c$ is the exposure constant characteristic of the photoresist, $r_{1}$ is the rate of etching of exposed molecules, $r_{2}$ is the rate

The authors are with California Institute of Technology, Pasadena, California 91125.

Received 27 November 1976. of etching of unexposed ones, and $\Delta r=\left(r_{1}-r_{2}\right)$. If the term $c E$ is much less than $1, \mathrm{Eq}$. (1) can be linearized as follows:

$$
\Delta d \sim \Delta r T c E+r_{2} T .
$$

In this work we determined the parameters involved in Eq. (2) for the Shipley AZ-1350B photoresist used with the AZ-303A developer.

The samples used were NO. 3010 microscope slides made by Clay Adams, which, cut in half, resulted in a size of $38 \mathrm{~mm} \times 25 \mathrm{~mm} \times 1 \mathrm{~mm}$. The samples were cleaned according to the method presented in Ref. 8 , and for some experiments the back surface was painted black with $3 \mathrm{M}$ Nextel 101-C10 velvet coating. The AZ-1350B photoresist was then deposited in a single layer, and after $30 \mathrm{sec}$ it was spun at $3600 \mathrm{rpm}$ for $30 \mathrm{sec}$. The samples were baked, next, for $30 \mathrm{~min}$ at $100^{\circ} \mathrm{C}$.

The first experiment involved the determination of the etch rate of the unexposed resist as a function of development time for various solutions of AZ-303A developer with distilled water. For this purpose the samples were half immersed in the developer for the required time, rinsed with deionized water for $2 \mathrm{~min}$, and then baked under vacuum at $100^{\circ} \mathrm{C}$ for $30 \mathrm{~min}$. The step size was measured using a Sloan Dektat instrument, and the results are shown in Fig. 1. The 4:1 solution (four parts distilled water, 1 part AZ-303A developer) gave unacceptably high etch rates, and the 8:1 gave low and nonlinear ones. We chose the 6:1 dilution for all our experiments, since it exhibited a linear behavior and an acceptable etch rate of $r_{2}=35$ $\AA \pm 5 \AA /$ sec. The development time chosen for the subsequent experiment was $10 \mathrm{sec}$.

For comparison, we performed the same experiment using the Shipley MF-312 developer. This developer is free from trace-metallic elements and is commonly used in the fabrication of photoresist gratings in semiconductor substrates. Figure 2 shows the unexposed etch rate as a function of development time in minutes for the manufacturer's recommended dilution of 1:1. In 


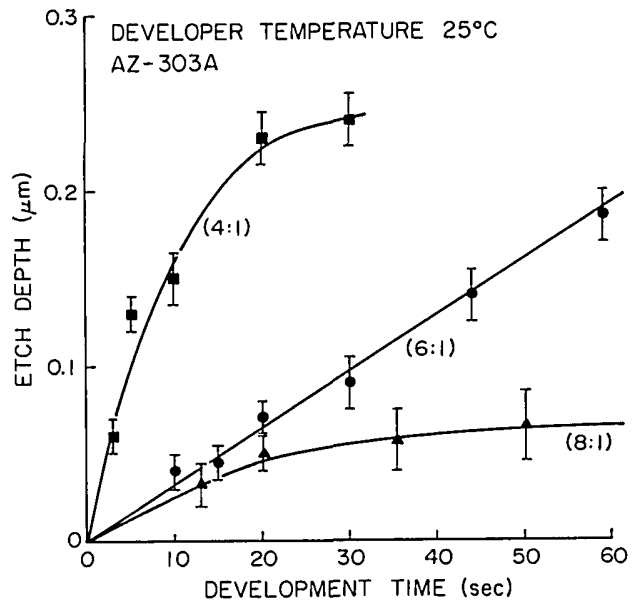

Fig. 1. Etch depths in $\mu \mathrm{m}$ of unexposed AZ-1350B photoresist as a function of development time in seconds for various dilutions ratios of AZ-303A developer. The slope of the curves determines $r_{2}$, which for the $6: 1$ dilution is $35 \AA \pm 5 \AA$.

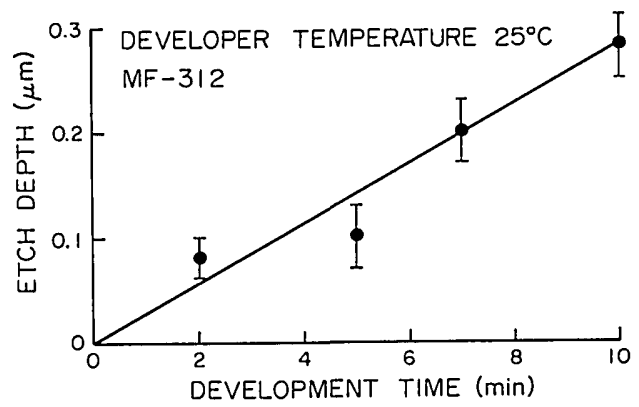

Fig. 2. Etch depth in $\mu \mathrm{m}$ of unexposed AZ-1350B photoresist as a function of development time in minutes for MF-312 developer. The 1:1 dilution results in $r_{2}=5 \AA \pm 1 \AA$.

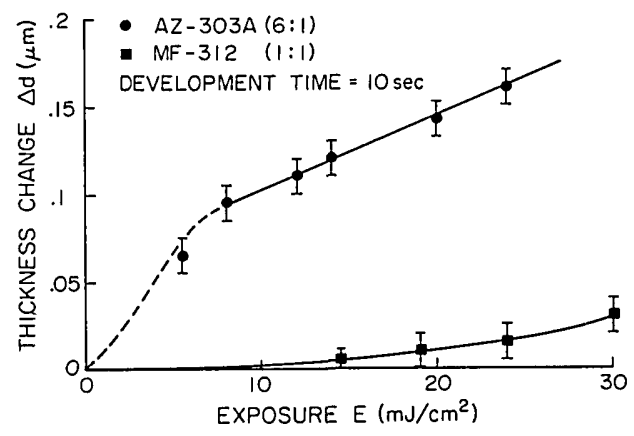

Fig. 3. Thickness change $\Delta d$ in $\mu \mathrm{m}$ of AZ-1350B photoresist as a function of exposure $E \mathrm{in} \mathrm{mW} / \mathrm{cm}^{2}$. The circles represent the AZ303A developer, and the squares represent the MF-312 developer.
Table 1. Unexposed Etch Rate for AZ Photoresists and AZ-303A Developer

\begin{tabular}{lccc}
\hline Photoresist & $\begin{array}{c}\text { Unexposed } \\
\text { etch rate }\end{array}$ & $\begin{array}{c}\text { AZ-303A } \\
\text { dilution }\end{array}$ & Reference \\
\hline $\begin{array}{l}\text { AZ-1350 } \\
\text { replaced by }\end{array}$ & $150 \AA / \mathrm{sec}$ & $4: 1$ & 4,5 \\
$\begin{array}{l}\text { AZ-1350B } \\
\begin{array}{c}\text { AZ-1350H } \\
\text { replaced by }\end{array}\end{array}$ & $35 \AA / \mathrm{sec}$ & $6: 1$ & This paper \\
AZ-1350J & $200 \AA / \mathrm{sec}$ & $6: 1$ & - \\
\hline
\end{tabular}

this case $r_{2}$ is $5 \AA \pm 1 \AA / \mathrm{sec}$, which is a much lower etch rate than the one for $\mathrm{AZ}-303 \mathrm{~A}$ developer. If resist thickness is small, if long exposure times are acceptable, and if linearity is unimportant, this may indeed be a better choice.

The results for the different resists using the different dilutions of the AZ-303A developer are given in Table I.

To demonstrate the thickness change as a function of exposure time, the $0.4416-\mu \mathrm{m}$ line of a He-Cd laser was used to illuminate half of the sample. The back surface of the sample was coated with black paint to avoid interference fringes. The intensity distribution of the laser was better than $5 \%$ across the surface of the sample. The photoresist was exposed for a given time and then developed $10 \mathrm{sec}$ in the AZ-303A developer 6:1 dilution. Figure 3 shows the thickness change as a function of exposure energy. It can be clearly seen that the behavior of the photoresist in the important 0.1$0.2-\mu \mathrm{m}$ range is linear. In contrast, the MF-312 developer gave very small thickness changes for the same range of exposures. In order to verify that the stylus of the instrument was not scratching the surface, the same samples were aluminized and then tested.

To demonstrate the feasibility of using AZ-1350B photoresist with the AZ-303A developer in making high efficiency gratings, the following experiment was performed. Photoresist was spin-coated on samples at $3000 \mathrm{rpm}$, resulting in a resist thickness of about $3.1 \mu \mathrm{m}$. The gratings were generated by exposing the samples to the sinusoidal intensity distribution produced by the interference pattern of two collimated $\mathrm{Ar}^{+}$laser beams. The wavelength used was $0.4579 \mu \mathrm{m}$, the angle between the beams $94.5^{\circ}$, and the intensity per beam 0.60 $\mathrm{mW} / \mathrm{cm}^{2}$. The exposed samples were developed in AZ-303A developer, baked under vacuum, and the efficiency of the gratings was measured. Figure 4 shows the absolute efficiency of the gratings as a function of exposure for two different development times. It is clear that high efficiency $23 \%$ resulted by developing the samples for $10 \mathrm{sec}$. To verify the theoretically predicted period and the peak to trough height, a scanning electron microscope was used. The period was measured to be $0.31 \mu \mathrm{m}$, and the peak to trough height was 0.28 $\mu \mathrm{m}$. 


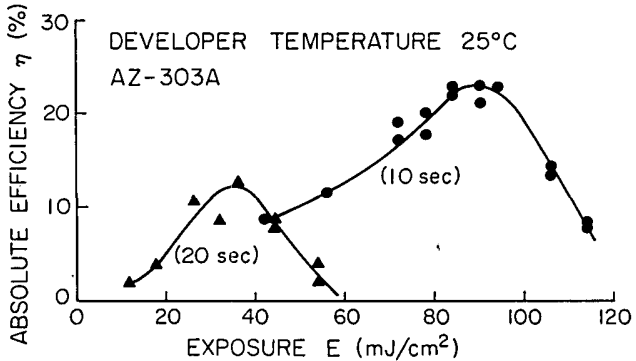

Fig. 4. Grating efficiency (absolute) as a function of exposure $E$ in $\mathrm{mW} / \mathrm{cm}^{2}$ for 10 -sec and 20 -sec development times in AZ-303A developer. Initial resist thickness was $0.31 \mu \mathrm{m}$.

In conclusion, we have found that the use of AZ-303A developer with the AZ-1350B photoresist results in an unexposed etch rate of $\sim 35 \AA / \mathrm{sec}$ and significantly increases the sensitivity and linearity of the photoresist in the $0.05-0.2-\mu \mathrm{m}$ range. We have successfully made gratings with constant and variable period ${ }^{9}$ using this method and have transferred them to glass using ion beam etching techniques.
The authors thank D. R. Armstrong for his valuable technical assistance. This research was supported by the U.S. Air Force Office of Scientific Research. The work of J. B. Shellan is supported in part by the Hertz Foundation.

\section{References}

1. H. L. Garvin, E. Garmire, S. Somekh, H. Stoll, and A. Yariv, Appl. Opt. 12, 455 (1973).

2. S. Austin and F. T. Stone, Appl. Opt. 15, 1071 (1976).

3. S. Austin and F. T. Stone, Appl. Opt. 15, 2126 (1976).

4. R. A. Bartolini, Appl. Opt. 11, 1275 (1972).

5. R. A. Bartolini, Appl. Opt. 13, 129 (1974).

6. M. S. Htoo, Photogr. Sci. Eng. 12, 169 (1968).

7. F. J. Loprest and E. A. Fitzgerald, Photogr. Sci. Eng. 15, 260 (1971).

8. S. L. Norman and M. P. Singh, Appl. Opt. 14, 818 (1975).

9. A. C. Livanos, A. Katzir, and A. Yariv, Opt. Commun. 20, 179 (1977).

\section{Uniformly spaced color samples}

The Optical Society of America announces the availability of a set of 552 uniformly spaced color samples designed by the OSA Committee on Uniform Color Scales. The colors can be arranged in hundreds of orderly sequences called color scales, most of which have never been seen previously because they cannot be constructed by use of any other set of color cards. Plans for arranging the cards in those scales will be provided.

Artists and designers have discovered that harmonious combinations result when a few colors selected from any such scale are used as elements of a design or composition. The OSA colors will reveal many new scales and suggest to creative workers, for their trial and selection, many new combinations of colors.

Although scales of constant lightness and constant hue are included, most of the scales exhibit variations of both attributes, in many different combinations. Each color card (except the darkest, or lightest, or those of highest saturation) is a member of six entirely different color scales. Most color scales do not include a neutral (black, gray, or white). For further information, see J. Opt. Soc. Am. 64, 1691 (1974).

The OSA color cards are two inches square, coated with durable, glossy automobile-finish paint on highest-quality heavy white card-stock paper. The OSA committee's three-number color designation-lightness, yellowness, and greenness-is printed on the back of each card. Negatives as well as positive values of any or all of those numbers provide designations for all possible colors. All colors producible with durable paints are represented in the set.
The set of cards is furnished in a 3-ring hardback notebook that holds 28 flexible, transparent pages; each page contains 20 pockets, into each of which one color card is inserted. Behind each pocket page is a white-paper guide page on which the three-number designation of the appropriate color card is printed so as to be visible through any pocket from which the color card has been removed for assembly into a color scale, or for any other use. The notebook can therefore be easily restored to its original state after selected cards have been removed for use.

Sets may be ordered from the Optical Society of America, Suite 620, 2000 L Street, N.W., Washington, D.C. 20036. The price is $\$ 350$. Include remittance with personal orders. A small number of sets of larger cards, without notebooks, is available on a first-come first-served basis. Some large cards have minor scratches on the surface and/or small flaws close to one edge. Prices (for complete sets only): slightly larger than $6 \times 8$ in. $\$ 4000$; slightly larger than $4 \times 6$ in. $\$ 2100$; slightly larger than $2 \times 6$ in. or $3 \times 4$ in., $\$ 1100$.

The production of this set of colors is the culmination of 35 years of research by members of the OSA committee directed toward producing a set of samples whose color differences consist solely of chromaticity differences without luminance differences. 